\title{
Reflections on Synesthesia, Perception, and Cognition
}

\author{
Noam SAGIV*, Alireza ILBEIGI*, Oded BEN-TAL
}

\begin{abstract}
In this paper, we reflect on three long-standing problems: The relationship between the physical world and the perceived world, accounting for individual differences in the way in which we perceive the world around us, and the problem of understanding other minds. We begin by examining the relationship between synaesthesia and hallucinations, as well as between hallucinations and normal perception, attempting to show in both cases that the phenomena in question may share more in common than previously assumed. We consider the plausibility of a functional analysis of synaesthesia and examine the mechanisms of the different types of ordinary and extraordinary perceptions. We propose that synaesthesia-like mechanisms may underlie a range of perceptual phenomena and cognitive functions and demonstrate the usefulness of such an approach given the ubiquity of synaesthesia-like mental processes in human cognition.
\end{abstract}

Key words: synesthesia, synaesthesia, hallucinations, visual perception, cognition, mental processes, sensory thresholds, neuropsychology, perceptual disorders.

RESUME. Réflexions sur la synesthésie, la perception et la cognition. Nous nous penchons dans cet article sur trois questions toujours actuelles : sur le rapport entre le monde physique et le monde perçu, sur la difficulté d'expliquer les différences individuelles dans la perception du monde environnant, et sur l'énigme de la compréhension de l'esprit d'autrui. En examinant la relation entre la synesthésie et les hallucinations, et entre les hallucinations et la perception normale, nous montrons que tous ces phénomènes ont bien plus en commun qu'on ne le supputait. Nous nous interrogeons ensuite sur la plausibilité d'une analyse fonctionnelle de la synesthésie, et examinons les mécanismes de différents types de perception ordinaire et extraordinaire. Nous soutenons que des mécanismes de même type que ceux impliqués dans les synesthésies pourraient intervenir tout un éventail de phénomènes perceptifs et cognitifs, et montrons l'utilité d'une telle approche eu égard à l'ubiquité dans la cognition humaine des processus qui formellement rappellent la synesthésie.

Mots clés : synesthésie, hallucinations, perception visuelle, cognition, processus mentaux, seuil sensoriels, neuropsychologie, troubles de la perception.

\section{INTRODUCTION}

We constantly receive signals from different sensory modalities and must combine those in order to understand objects and events around us (Macaluso \& Driver, 2005). Hence, there is little wonder that substantial cross-modal interactions take place in the human brain. What is more surprising is that in a minority of individuals such interactions occur when a stimulus is presented in only one sensory modality. This is the case in synaesthesia - where perceptual experience normally associated with one sensory modality may be elicited by stimuli presented in another modality (e.g., Sagiv, 2005; Ward, 2008; Cytowic \& Eagleman, 2009). For example, colour experience induced by sounds or

" Centre for Cognition and Neuroimaging, Department of Psychology, Brunel University, West London, UB8 3PH, United Kingdom. Address correspondence to Noam Sagiv.

" Music Department, Kingston University, Kingston-upon-Thames, KT2 7LB, United Kingdom. 
smells. However synaesthesia can also be induced by ordinal sequences: It is fairly common among synaesthetes to associate letters and numbers (presented in black and white) with specific colours (e.g., Cohen-Kadosh et al., 2005) that are vividly perceived either 'in the mind's eye' or projected externally - usually onto the letter surface (Dixon, Smilek, \& Merikle, 2004; Ward, Li, Salih, \& Sagiv, 2007) ${ }^{1}$.

Although previously thought to be a rather rare condition, synaesthesia is present in at least 4\% of the general population (Simner et al., 2006). Unlike other types of mental imagery, synaesthetic imagery is induced automatically (e.g., Rich et al., 2006). Unlike hallucinations, synaesthetic images are often very predictable: Synaesthetic correspondences tend to be consistently evoked by certain stimuli across long periods of time in each individual (e.g., Van Campen, 2007; Cytowic \& Eaglman, 2009). This has made it easier for scientists to study the phenomenon. Furthermore, the permanence and regularity of synaesthetic experiences also means that for the synaesthete, these experiences are an integral part of everyday experience ${ }^{2}$. Most of us - both synaesthetes and non-synaesthetes - take our perceptual experiences for granted, and rarely consider that others' experiences may be very different. Synaesthesia may appear odd to non-synaesthetes, but synaesthetes are just as surprised to hear that the rest of us do not experience synaesthesia (e.g., Sagiv, Heer, \& Robertson, 2006).

Synaesthesia has often been conceptualised as an abnormality (e.g., "breakdown of modularity"; Baron-Cohen, Harrison, Goldstein, \& Wyke, 1993). However, in the past decade, we have discovered that synaesthesia shares much in common with ordinary perception; it relies on common perceptual mechanisms and may merely represent an augmentation of normal propensities for cross-modal interactions (Sagiv \& Ward, 2006). But how different is synaesthesia from ordinary perception and from other forms of extraordinary perception?

In the present paper, we reflect about the relationship between synaesthesia, hallucinations, and ordinary ('normal' or veridical) perception. We shall try to convince the reader that these may share more in common than previously assumed. We will then assess whether synaesthesia could be incorporated into a functionalist framework. Special consideration is given to the problem of accounting for individual differences. Furthermore, we will show that synaesthesia-like mechanisms may underlie a range of perceptual phenomena and cognitive functions.

\section{SYNAESTHESIA AND HALLUCINATIONS}

The perceptual experiences of individuals with synaesthesia do not seem to match what most of us will describe under similar circumstances. For example, perceiving colours that are not really there, sounds that cannot be heard by others, or smells that cannot be explained by the presence of any airborne molecules. It is easy to see that such a description does actually satisfy the

\footnotetext{
${ }^{1}$ In such a case synaesthesia may involve two different sub-modalities or two different features within one modality (e.g., letter shape and colour).

${ }^{2}$ For an extensive discussion of the personal, social, emotional and aesthetic aspects, see Van Campen (2007) or Dittmar (2009).
} 
basic requirement set in common definitions of hallucinations ${ }^{3}$. One of the criteria in Slade and Bentall's (1988) suggested definition of hallucinations is that the experience "occurs in the absence of an appropriate stimulus". At first glance, this seems like a very straightforward requirement. Take for example the case of seeing a cat. Seeing one when no stimulus is presented is of course a hallucination. But seeing a cat when looking at a mouse is also a case in which the appropriate stimulus (a cat) is absent. Another instance is seeing a cat when the only stimulus present is the sound of birds singing (again - the cat is absent). Are these all hallucinations? In practice, neuropsychologists do distinguish between spontaneous hallucinations and misperception of external stimuli; the latter falls under the broad category of 'metamorphopsia' (at least when referring to visual stimuli, as indicated by the suffix). This includes a range of phenomena in which a stimulus may be perceived as distorted in various ways or misidentified altogether ${ }^{4}$.

Could hallucinations be triggered by a stimulus in a different modality? The DSM-IV definition implicitly acknowledges this possibility by defining hallucination as a sensory perception that "occurs without external stimulation of the relevant sensory modality". If external stimulation is presented only in what most people would describe as an irrelevant modality (different than the one experienced), those who adhere to the DSM definition would have to conclude that synaesthesia could be regarded as a special case of hallucination. It is not our intention, however, to suggest that synaesthesia should be regarded as a pathological condition ${ }^{5}$. Admittedly, there are many differences between developmental synaesthesia and spontaneous hallucination: Developmental synaesthesia is not transient; it is elicited in a consistent manner by specific stimuli; it is not disruptive; and it occurs naturally without neurological disease or the aid of recreational drugs (Van Campen, 2007).

What we are suggesting is that there is a simple common characteristic between synaesthesia and hallucination: The absence of an 'appropriate' stimulus. Of course, we use the term appropriate in a statistical sense only (the appropriate stimulus is what the majority of people consider a plausible inducer of the perceptual experience in question). Therefore we can distinguish between three classes of perceptual phenomena occurring without an 'appropriate' stimulus: Spontaneous hallucination (with no obvious external trigger); metamorphopsia (triggered by a stimulus in the same modality), and synaesthetic hallucinations (triggered by a stimulus in a different modality). While in principle, the experience itself may be very similar, what is different here is the trigger. The underlying mechanisms may or may not be similar - we return to this point later. All of these are of course the consequence of the neurophysiology and the neural architecture, whether intact, damaged, or just atypical).

3 See Shanon (2003) for an extended discussion of various suggested definitions of hallucinations. 4 For example, a human face misperceived as dog face (Grüsser \& Landis, 1991); this is a specific type of metamorphopsia labelled prosopometamorphopsia. It is clearly of central origin (though note that some simpler distortions can be due to lower-level eye conditions). Unlike common visual illusions we are all susceptible to, higher level distortions in metamorphopsia can be idiosyncratic and may result from brain damage.

5 The purpose of examining definitions (and their loopholes) is to reflect on our conceptualisation of a range of perceptual phenomena and analyse similarities and differences between them. We urge readers with strong feelings against viewing synaesthesia as an instance of hallucination to bear with us and withhold judgment for now. 
While synaesthesia researchers have carefully analysed the differences between synaesthesia and spontaneous hallucination, we find it conceivable that some surveys of hallucinations have lumped together spontaneous hallucinations and synaesthetic hallucinations where no consideration has been given to whether or not there was a trigger and to which modality it belonged (Sagiv \& Ben-Tal, submitted). Take for example the case of hypnagogic hallucinations: Instances of synaesthesia between sleep and wakefulness have been documented in several studies (Bachem, 1949; Hollingwoth, 1911; Podoll \& Robinson, 2002; Vihvelin, 1948) but there usually is no mention of these in large scale surveys of hallucinations in the general population although hypnagogic/hypnopompic hallucinations are the most frequently reported hallucinations in the general population (e.g., Ohayon, 2000).

In sum, we have seen that synaesthetic experience may have at least one common feature with spontaneous hallucinations. We feel that acknowledging this is necessary if we are going to try to develop a unified framework for understanding a range of (ordinary and) extraordinary perceptual experiences. Similarly, it would be helpful to point out similarities between hallucinations and normal perception. We recognise that hallucinations are traditionally seen in a negative light; synaesthesia researchers and synaesthetes alike have often tried to distance themselves from the topic. Thus, our next aim is to briefly survey what hallucinations share with ordinary perceptual experiences, perhaps dispelling at least some of the negative views about hallucination.

\section{Hallucinations and Normal Perception}

There are various patient groups that are particularly prone to hallucinate; for example, patients with sensory loss (e.g., Charles Bonnet Syndrome; ffytche, 2005), psychiatric patients (Bentall, 1990), and patients with temporal lobe epilepsy (Taylor \& Lochery, 1987), to name a few conditions. However, hallucinations are also reported by healthy individuals between sleep and wakefulness (e.g., Ohayon, 2000) or under the influence of hallucinogenic drugs (Klüver, 1966). What is common to all of these examples is a weaker correspondence between the perceived world and the actual sensory stimulation. The term 'pseudo-hallucination' is sometimes used to describe the condition of patients who are aware that what they perceive is not real (this is common in hallucinations linked with deafferentation, for example, Charles Bonnet patients are not delusional and usually distinguish between veridical percepts and their hallucinations; ffytche, 2005). It is not clear, however, that there is a fail-safe way to distinguish all hallucination from veridical perception. It is often the improbability of the perceptual experience that gives away hallucinations (e.g., seeing a racoon in the library) but if one were to hallucinate more probable sights (e.g., an extra bookshelf in the library) this may go unnoticed.

Therefore, the boundary between veridical perception and various forms of misperceptions and hallucinations is not as clear cut as it may seem - at least when considering the phenomenology. All of these perceptual experiences are constructs of the mind; while what we perceive is usually highly constrained by the physical environment, hallucinations are merely under-constrained percepts (Behrendt and Young, 2004) ${ }^{6}$. Thus, deviations from so-called normal

\footnotetext{
${ }^{6}$ According to Behrendt and Young (2004), under-constrained percepts occur when sensory inputs have a reduced impact on thalamocortical circuit activity. This may be the result of a combination of factors
} 
perception manifest as a mismatch between the perceived world and the physical world, but such deviations do not represent a categorically different type of perceptual experience.

This view is consistent with Dennett's (1991) claims that our perceptual experiences should not be regarded as some sort of a movie in the brain but rather as a series of judgments we are making. According to this view hallucinations may be understood simply as incorrect judgments. Similarly, Gregory (1980) likened perceptions to hypotheses. To see an object in front of us is akin to hypothesizing that it is there. The hypothesis may turn out to be true or false $^{7}-\mathrm{a}$ hit or a false alarm, respectively, using Signal Detection Theory terminology.

Where does synaesthesia fit within the framework? The case of developmental synaesthesia is interesting because perception is constrained by the sensory input in the sense that specific synaesthetic experiences are predictably elicited by specific stimuli. In other words - although the correspondence between sensory input and perceptual experience is different in synaesthetes and non-synaesthetes - in both groups this correspondence is regular. Furthermore, this regularity is something that synaesthetes can rely on to make sense of the world around them. For example, a synaesthete could distinguish between a bassoon and an oboe sound not only because they sound different, but also because they have their own unique colours. In that sense, synaesthesia is closer to veridical perception than to hallucinations. Furthermore, synaesthetic experience has a predictive value concerning the likely source and could therefore serve a useful function.

Nevertheless, to analyse synaesthesia in functional terms, we must deal with several challenges. One challenge is to account for the somewhat arbitrary nature of synaesthetic correspondences. Synaesthetic images may make sense for each synaesthete, but in the eyes of any other synaesthetes (or a nonsynaesthete for that matter), the reported experiences may appear to lack correspondence with the sensory input.

A second question is whether it is possible or meaningful to compare experiences between individuals? Should a synaesthete hear the sound of a trumpet and make the correct inference concerning its source, does it matter at all that his or her experience may be different than that of a non-synaesthete who listens to the same trumpet sound but does not see any colours while the recital goes on? What is clear, however, is that a purely behavioural functional analysis cannot explain how the same simple stimulus in a given context could evoke two qualitatively different experiences in different individuals as pointed out by Gray et al. (2002).

such as a cortical release mechanism in sensory loss or sensory deprivation, cortical irritation, cortical hyperexcitability, and more (Collerton, Perry, \& McKeith, 2005).

${ }^{7}$ Note that the hypothesis we are talking about, refers to the pre-reflective, raw experience. Upon reflection, we may come to the conclusion that our visual impression (e.g., a unicorn or a golden cityscape) cannot be an accurate portrayal of reality; otherwise, it may be up to independent observers to judge. 


\section{Is a Functionalist Analysis of Synaesthesia Possible?}

Can synaesthesia be accommodated by a functionalist framework? As we have seen - although some synaesthetic correspondences are idiosyncratic, the perceptual experience can be identified with certain functional states, for the individual concerned. According to Gray et al. (2002), a functionalist would explain synaesthesia as resulting from associative learning, while brain imaging findings support a different explanation, namely, the hyperconnectivity of certain parts of the cerebral cortex. This left us wondering why we must assume that the only functional explanation involves associative learning. If we accept that synaesthesia is the consequence of anomalous brain connectivity, why can't this be analysed functionally? After all - any theory of how a system works must also incorporate predictions on possible 'dysfunctions' when certain parameters deviate from the norm. Therefore, the presence of an anomaly does not rule out a functional explanation. Indeed, Noe and Hurley (2003) do incorporate neural activity into their framework for understanding the qualitative nature of perceptual experience (within the broader dynamic sensorimotor context).

While it appears that synaesthetes do indeed exhibit such hyperconnectivity (Rouw \& Scholte, 2007) to which they are likely to be genetically predisposed (Asher et al, 2009), it seems clear that some learning has to take place in those predisposed individuals in order to determine, at the very least, the specific cross-modal correspondences unique to each synaesthete (see Hunt, 2005, for an extended discussion of the role of learning in synaesthesia). The biggest challenge synaesthesia poses to functionalism (and in fact, any framework for understanding consciousness) is perhaps the idiosyncratic nature of some synaesthetic correspondences. To account for these it may be necessary to obtain very detailed data on environmental factors and learning during the course of development. We suspect that the neuroanatomical and behavioural accounts of synaesthesia may be reconciled if we take development seriously and recognise that such a complex phenomenon, like many others, must be the product of a dynamic interaction between neurophysiological, genetic, and environmental constraints.

While the ultimate goal is to account for specific mapping between inducing stimuli and the evoked experiences, it has proved easier to explain why certain individuals have a particular type of synaesthesia and not another. Indeed, much progress has been made in understanding the neural mechanisms underlying both ordinary and extraordinary perception, including synaesthesia, hallucinations, and other positive symptoms. In the final three sections we focus on the neurobiological level, compare and contrast the mechanisms of (i) synaesthesia and hallucinations, (ii) synaesthesia and other positive perceptual symptoms, (iii) synaesthesia and normal perception.

\section{THE MECHANiSMS OF SyNAESTHESIA AND HALLUCINATION}

It is widely accepted that synaesthesia could result from cross-activation of different sensory areas in the brain (Ramachandran \& Hubbard, 2001). Functional neuroimaging data is consistent with this claim, although the exact nature of the underlying mechanism is still under debate. There is compelling evidence for anatomical differences between (developmental) synaesthetes and non-synaesthetes. Diffusion tensor imaging (DTI) confirms the predicted 
cortical hyperconnectivity involving the fusiform gyrus in synaesthetes (Rouw \& Scholte, 2007), but it is not clear that hyperconnectivity could account for all instances of synaesthesia, especially when induced temporarily in nonsynaesthetes, i.e., with hallucinogens or hypnosis. The latter case has been attributed to disinhibition (Cohen-Kadosh et al., 2009) - indeed it is unlikely that substantial changes in cortico-cortical connectivity have occurred over short timescale in the case of hypnosis-induced synaesthesia.

Given a neural configuration with either hyperconnectivity or lower thresholds for excitation (whether pharmacologically induced, due to sensory deprivation, or to hypnosis) a uni-modal stimulus may indeed elicit an experience in more than one sensory modality. In such a case, it would not make sense to talk about the absence of an 'appropriate' stimulus because we understand very well why an experience in an additional modality should in fact be expected. Given a perceptual experience, we could conceivably refine the definition of 'appropriate stimulus' to account for individual differences, and tailor the range of what we describe as appropriate stimuli to the neural architecture. After all, there is no one-to-one correspondence between stimulus modality and the modality of the experience it gives rise to, and neural constraints must be taken into consideration (for example, if there is damage to the optic nerve, visual stimuli may not elicit a visual experience at all). This exposes a circularity in the definition of hallucinations as lacking appropriate stimuli: The more we understand about the neural basis of perception, the less surprised we should be that certain individuals' experiences are richer than others'. Eventually, it boils down to our tolerance to deviation in the norm, whether it is at the neural, behavioural, or experiential level.

Now that we have discussed the neurobiological variability, let us examine what are some of the common neural mechanisms of synaesthesia, hallucinations, and ordinary perceptual experiences. Colour experience, for example, seems to consistently involve some of the same visual areas whether triggered by the range of light wavelengths falling on the retina (Bartels \& Zeki, 2000), spontaneously arising in Charles Bonnet hallucinations (ffytche et al., 1998), or triggered by an auditory stimulus in synaesthetes (Nunn et al, 2002).

In both synaesthesia and hallucination activation of cortical colour processing areas occurs without the sensory input normally associated with colour. In synaesthesia, it is the result of co-activation when processing another stimulus. In hallucination, there is no stimulus - or so it seems. Most certainly, the cortical activations we see in hallucination are causally related to the neural events immediately preceding the onset of hallucinations. We might therefore ask whether hallucinations could result from a synaesthesia-like cross-activation of brain areas? The trigger of the hallucinatory experience may not be obvious; in fact, it may be internal rather than an external stimulus. A number of different mechanisms may be responsible for lower thresholds for activation that makes one susceptible to hallucinations (e.g., drugs, deafferentation or temporary sensory deprivation). Given that the thresholds for activation of specific cortical areas are lower than usual in both synaesthesia and hallucinations, it is possible that better understanding of genetic and epigenetic factors in synaesthesia, may yield some insights into why certain individuals are more susceptible to hallucinations than others. It is an open question whether synaesthesia has anything to do with susceptibility to hallucinations, but it should be easy to test. If there is such a link, we would expect, for example, that in a large sample of individuals with eye disease (a risk factor for visual hallucina- 
tion), those who have colour synaesthesia, will also be more susceptible to spontaneous colour hallucination than non-synaesthetes (or taste synaesthetes for that matter). Another prediction is that using transcranial magnetic stimulation (TMS), phosphenes could be induced at lower thresholds in those with visual synaesthetic experiences, than in those with tactile synaesthetic experiences.

We should also strive to characterise better the phenomenology of patients with hallucination. It may turn out that they experience more synaesthetic hallucinations than currently reported in the medical literature. Further exploration of the link between voluntary and involuntary forms of mental imagery will also be necessary if we are to understand the full range of perceptual experience. Indeed recent studies report enhanced mental imagery in synaesthetes (Barnett \& Newell, 2008; Price, 2009). Other studies show that synaesthetic experiences can be triggered by mental images of the usual inducing stimulus as well (Spiller \& Jansari, 2009). We speculate that a broad spectrum of human experiences must involve some sort of synaesthesia-like cross activation. After all, non-trivial mental function quite commonly requires the integration of information across different domains of perception, cognition, and action.

At the very least, the cross-activation account can be extended to types of synaesthesia where the trigger is not strictly sensory. For instance, quite commonly, the inducers of synaesthesia are concepts such as numbers (e.g., Sagiv, Heer, \& Robertson, 2006; Ward \& Sagiv, 2007) or days of the week (Shanon, 1982; Rich, Bradshaw, \& Mattingley, 2005; Simner et al., 2006). Similarly, in other synaesthesia variants (or synaesthesia-like phenomena if you wish) ${ }^{8}$, the induced experience may not be a simple sensory one. For example, 'number forms' - a visuo-spatial variant of synaesthesia - where numbers are associated with a shift of spatial attention to a particular location in space (Sagiv, Simner, Collins, Butterworth, \& Ward, 2006; Tang, Ward, and Butterworth, 2008). Another example is the personification of letters, numbers or objects (Day, 2005; Cytowic, 2002; Simner \& Holenstein, 2007; Smilek et al, 2007). It has been suggested that the cross-activation seen in more straightforward examples of synaesthesia could explain the more unusual variants as well (e.g., Simner \& Hubbard, 2006).

\section{SYNAESTHESIA AND OTHER POSITIVE SYMPTOMS}

Can these ideas be extended to other positive symptoms? A synaesthesialike mechanism was suggested for autoscopy (Zamboni, Budriesi, \& Nichelli, 2005) - a rare condition in which the patient hallucinates a mirror image of his or herself. Autoscopy belongs to a class of phenomena called autoscopic phenomena also including (1) Out-of-body experience (e.g., Blanke \& Mohr, 2005); (2) Doppelgänger or double delusions (e.g., Brugger, 2002); and (3)

\footnotetext{
${ }^{8}$ Although there is no consensus on whether grapheme personification and number forms should be regarded as types of synaesthesia, they do share some phenomenological, behavioural, neurobiological and likely also genetic features. Indeed, with dozens of types of synaesthesia documented in the literature, it has proved quite challenging to agree on a definition for synaesthesia or a set of behavioural criteria to diagnose it. The definition of synaesthesia is not an empirical question, but it should be informed by phenomenology, behavioural, and neurobiological data (Sagiv, 2008). Indeed Simner (in press) has recently echoed this and called to move towards a neurobiological definition rather than relying on a purely behavioural diagnosis.
} 
sense of presence, reported for example in bereavement (Rees, 1971) or when the temporo-parietal junction is stimulated (Arzy, Seeck, Ortigue, Spinelli, \& Blanke, 2006). What is common to all of this is mirroring of one's own body or self or projecting it outside the body. The body image as it is constructed and perceived requires the integration of multisensory information including at the very least proprioceptive, haptic, and visual information. Mismatch or excessive cross-activations between those different sources of information can therefore yield a range of embodiment illusions whether the anomalous interactions are due to a neurological condition (Blanke, Landis, Spinelli, \& Seeck, 2004), are induced deliberately in the laboratory (e.g., the rubber hand illusion, Botvinick \& Cohen, 1998) or correspond to whole body illusions induced with a virtual reality apparatus (e.g., Lenggenhager, Tadi, Metzinger, \& Blanke, 2007). Explaining phenomena implicating alterations to the body image or the body schema ${ }^{9}$ based on a cross-activation mechanism similar to the one we see in synaesthesia, seems like a promising direction, especially when sensory inputs are manipulated. Recall for example Ramachandran and RogersRamachandran's (1996) work on restoring phantom limb mobility by providing visual input using a mirror.

While in autoscopic phenomena, the body may be visually mirrored or projected, the opposite occurs in 'mirror-touch synaesthesia'. Individuals with mirror-touch synaesthesia project visually presented tactile stimulation of others onto their selves, resulting in a tactile experience (Banissy, Cohen-Kadosh, Maus, Walsh, \& Ward, 2009); i.e., they can actually feel on their own body what other people are feeling when they are touched. This form of synaesthesia is an intriguing one since unlike other variants of synaesthesia, here the synaesthetic correspondences are not idiosyncratic at all but rather regular and constrained by the evoking stimulus. Furthermore, over-activation of the mirror neuron system beyond the threshold of conscious tactile experience appears to underlie the condition (Blakemore, Bristow, Bird, Frith, \& Ward, 2005). Applied to the visuo-tactile domains, the cross-activation theory of synaesthesia yet again offers a plausible explanation.

\section{BACK TO 'NORMAL' COGNITION}

Multisensory integration including the visuo-tactile interaction we see in mirror-touch synaesthesia does indeed enable a range of human skills; specifically, understanding people's intentions, feelings, and emotional state. It has been suggested that these important social skills cannot be based solely on abstract reasoning about other people's mental lives; instead, these skills rely on taking the other's perspective by simulating their actions using mirror neurons (Gallese \& Goldman, 1998; Gallese, 2007). Social cognition in most cases involves actually sharing experiences; to some extent we can actually feel other people's pain, sadness or happiness. Simulated experiences allow a quick, intuitive and direct appreciation of other minds that is essential for social interaction. If we do simulate other people's intentions and feelings - this could be conceptualised as a sensory-motor or sensory-sensory synaesthesia-like mapping of others' mental states onto ourselves; consider for example overt manifestations such as the subtle automatic mimicking of facial expression (e.g., Dimberg, 1982) - where the visual input concerning another person's state

${ }^{9}$ For a discussion of the distinction between body image and body schema, see De Vignemont (2009). 
activates the corresponding motor plan in the observer. Another example is the unpleasant experience of witnessing that a person we are close to is in pain. Again, we may have only witnessed the other person's facial expression (a visual trigger) or their voice (an auditory trigger) and yet our own experience may extend to the somatosensory domain. We propose that embodied social cognition may be best understood as a synaesthesia-like process. This point of view on the problem may generate novel hypotheses concerning the underlying cognitive, neural, and genetic basis of social cognition.

Cross-modal and cross-domain associations are indeed inherent to human cognition. We must constantly integrate information from different modalities and domains as well as switch between different frames of reference in order to make sense of objects and events around us. Moreover, we find it useful to deliberately translate data into a specific modality in order to extract information from it, e.g., visualisation and sonification of data (Ben-Tal \& Berger, 2004). Similarly, artists are able to utilise prevalent cross-modal associations as well as novel ones in order to create new forms of art (Sagiv, Dean, \& Bailes, 2009; van Campen, 2007); projecting and shifting from one reference frame to another (whether spatial, temporal, object- or person based) is also a fundamental feature of human cognition enabling a range of activities from enjoying literature, through driving, to planning our future.

We hope we have convinced the reader that synaesthesia provides an interesting test case for frameworks for understanding perception. Furthermore synaesthesia is but one of many mental processes involving cross-modal and cross-domain interactions and that studying synaesthesia may well generate novel insights into other aspects of human cognition in the foreseeable future.

\section{Acknowledgements}

Some of the ideas discussed here were first presented in the American Synesthesia Association meeting at McMaster University (2008), with support from a British Academy Overseas Conference Grant to NS. We thank David Tsabar for helpful comments on the manuscript.

\section{REFERENCES}

Arzy, S., Seeck, M., Ortigue, S., Spinelli, L., \& Blanke, O. (2006). Induction of an illusory shadow person. Nature, 443, 287.

Asher, J.E., Lamb, J.A., Brocklebank, D., Cazier, J.B., Maestrini, E., Addis, L., Sen, M., Baron-Cohen, S., \& Monaco, A.P. (2009). A whole-genome scan and finemapping linkage study of auditory-visual synesthesia reveals evidence of linkage to chromosomes 2q24, 5q33, 6p12, and 12p12. American Journal of Human Genetics, 84, 279-85.

Bachem, A. (1949). A new auditory-visual synesthesia. Acta Psychologica, 6, 363-364.

Banissy, M.J., Cohen Kadosh, R., Maus, G., Walsh, V., \& Ward, J. (2009). Prevalence, characteristics and a neurocognitive model of mirror-touch synaesthesia. Experimental Brain Research, 198, 261-272.

Baron-Cohen, S., Harrison, J., Goldstein, L.H., \& Wyke, M. (1993). Coloured speech perception: is synaesthesia what happens when modularity breaks down? Perception, 22, 419-26.

Barnett, K.J., \& Newell, F.N. (2008). Synaesthesia is associated with enhanced, selfrated visual imagery. Consciousness and Cognition, 17, 1032-9. 
Bartels, A., \& Zeki, S. (2000). The architecture of the colour centre in the human visual brain: new results and a review. European Journal of Neuroscience, 12, 172-93.

Behrendt, R.P., \& Young, C. (2004). Hallucinations in schizophrenia, sensory impairment, and brain disease: a unifying model. Behavioral and Brain Sciences, 27, 771-87.

Ben-Tal, O., \& Bereger, J. (2004). Creative Aspects of Sonification. Leonardo, 37, 229-233.

Bentall, R.P. (1990). The illusion of reality: a review and integration of psychological research on hallucinations. Psychological Bulletin, 107, 82-95.

Blakemore, S.J., Bristow, D., Bird, G., Frith, C., \& Ward, J. (2005). Somatosensory activations following the observation of touch and a case of vision-touch synaesthesia. Brain, 128, 1571-1583.

Blanke, O., Landis, T., Spinelli, L., \& Seeck, M. (2004). Out-of-body experience and autoscopy of neurological origin. Brain, 127, 243-58.

Blanke, O., \& Mohr, C. (2005). Out-of-body experience, heautoscopy, and autoscopic hallucination of neurological origin Implications for neurocognitive mechanisms of corporeal awareness and self-consciousness. Brain Research Reviews, 50, 184-99.

Botvinick, M., \& Cohen, J. (1998). Rubber hands 'feel' touch that eyes see. Nature, $391,756$.

Brugger, P. (2002). Reflective mirrors: perspective-taking in autoscopic phenomena. Cognitive Neuropsychiatry, 7, 179-94.

Cohen Kadosh, R., Henik, A., Catena, A., Walsh, V., \& Fuentes, L.J. (2009). Induced Cross-Modal Synaesthetic Experience Without Abnormal Neuronal Connections. Psychological Science, 20, 258-265.

Cohen Kadosh, R., Sagiv, N., Linden, D.E.J., Robertson, L.C., Elinger, G., \& Henik, A. (2005). When Blue is Larger than Red: Colors Influence Numerical Cognition in Synesthesia. Journal of Cognitive Neuroscience, 17, 1766-1773.

Collerton, D., Perry, E., \& McKeith, I. (2005). Why people see things that are not there: A novel Perception and Attention Deficit model for recurrent complex visual hallucinations. Behavioral and Brain Sciences, 28, 737-757

Cytowic, R. E. (2002). Synaesthesia: A Union of the Senses (2nd Edition). Cambridge, MA: MIT Press.

Cytowic, R.E., \& Eaglman, D. (2009). Wednesday is Indigo Blue: Discovering the Brain of Synesthesia. Cambridge, MA: MIT Press.

Day, S. (2005). Some demographic and socio-cultural aspects of synesthesia. In: L.C. Robertson \& N. Sagiv (Eds.), Synesthesia: Perspectives from Cognitive Neuroscience (pp 11-33). New York: Oxford University Press.

Dennett, D.C. (1991). Consciousness Explained. Boston, MA: Little, Brown \& Co.

de Vignemont, F. (2010). Body schema and body image - Pros and cons. Neuropsychologia , 48, 669-680.

Dimberg, U. (1982). Facial reactions to facial expressions. Psychophysiology, 19, 643647.

Dittmar, A. (2009). Synaesthesia: A "golden thread" through life? Essen: Die Blaue Eule.

Dixon, M.J., Smilek, D., \& Merikle, P.M. (2004). Not all synaesthetes are created equal: projector versus associator synaesthetes. Cognitive Affective and Behavioral Neuroscience, 4, 335-43.

Ffytche, D.H. (2005). Visual hallucinations and the Charles Bonnet syndrome. Current Psychiatry Reports., 7, 168-79. 
ffytche, D.H., Howard R.J., Brammer, M.J., David, A., Woodruff, P., Williams, S. (1998). The anatomy of conscious vision: an fMRI study of visual hallucinations . Nature Neuroscience, 1, 738-42.

Gallese, V. (2007). Before and below 'theory of mind': embodied simulation and the neural correlates of social cognition. Philosophical Transactions of the Royal Society B: Biological Sciences, 362, 659-69.

Gallese, V., \& Goldman, A. (1998). Mirror neurons and the simulation theory of mindreading. Trends in Cognitive Sciences, 2, 493-501.

Gray, J.A., Chopping, S., Nunn, J., Parslow, D., Gregory, L., Williams, S., Brammer, M.J., \& Baron-Cohen, S. (2002). Implications of synaesthesia for functionalism: Theory and Experiments. Journal of Consciousness Studies, 9, 5-31.

Gregory, R.L. (1980). Perceptions as hypotheses. Philosophical Transactions of the Royal Society B: Biological Sciences, 290, 181-97.

Grüsser, O. J., \& Landis, T. (1991). Visual agnosias and other disturbances of visual perception and cognition. In Vision and visual dysfunction (Vol. 12). London: Macmillan Press.

Hollingwoth, H. (1911). The psychology of drowsiness. American Journal of Psychology, 22, 99-111.

Hunt, H.T. (2005). Synaesthesia, Metaphor and Consciousness: A CognitiveDevelopmental Perspective. Journal of Consciousness Studies, 12, 26-45.

Klüver, H. (1966). Mescal and the Mechanisms of Hallucination. Chicago, IL: University of Chicago Press.

Lenggenhager, B., Tadi, T., Metzinger, T., \& Blanke, O. (2007). Video ergo sum: manipulating bodily self-consciousness. Science, 317, 1096-9.

Macaluso, E., \& Driver, J. (2005) Multisensory spatial interactions: a window onto functional integration in the human brain. Trends in Neurosciences, 28, 264-271.

Merleau-Ponty, M. (1945/1962). Phenomenology of Perception. New York: Routledge \& Kegan Paul.

Noë, A., \& Hurley, S. (2003). The deferential brain in action: Response to Jeffrey Gray. Trends in Cognitive Sciences, 7, 195-196.

Nunn, J.A., Gregory, L.J., Brammer, M., Williams, S.C., Parslow, D.M., Morgan, M.J., Morris, R.G., Bullmore, E.T., Baron-Cohen, S., \& Gray, J.A. (2002). Functional magnetic resonance imaging of synesthesia: activation of V4/V8 by spoken words. Nature Neuroscience, 5, 371-375.

Ohayon, M.M. (2000). Prevalence of hallucinations and their pathological associations in the general population. Psychiatry Research, 97, 153-64.

Price, M.C. (2009). Spatial forms and mental imagery. Cortex, 45, 1229-1245.

Podoll, K., \& Robinson, D. (2002). Auditory-visual synaesthesia in a patient with basilar migraine. Journal of Neurology, 249, 476-477.

Ramachandran, V.S., \& Hubbard, E.M. (2001). Synaesthesia - A Window Into Perception, Thought and Language. Journal of Consciousness Studies, 8, 3-34.

Ramachandran, V.S., \& Rogers-Ramachandran, D. (1996). Synaesthesia in phantom limbs induced with mirrors. Proceedings of the Royal Society of London B, 263, $377-386$

Rees, W.D. (1971). The Hallucinations of Widowhood. British Medical Journal, 4, 3741.

Rich, A.N., Bradshaw, J.L., \& Mattingley, J.B. (2005). A systematic, large-scale study of synaesthesia: implications for the role of early experience in lexical-colour associations. Cognition, 98, 53-84. 
Rich, A.N., Williams, M.A., Puce, A., Syngeniotis, A., Howard, M.A., McGlone, F., \& Mattingley J.B. (2006). Neural correlates of imagined and synaesthetic colours. Neuropsychologia, 44, 2918-25.

Rouw, R., \& Scholte, H. S. (2007). Increased structural connectivity in grapheme-color synesthesia. Nature Neuroscience, 10, 792-797.

Sagiv, N. (2005). Synesthesia in perspective. In: L.C. Robertson \& N. Sagiv (Eds.), Synesthesia: Perspectives from Cognitive Neuroscience (pp. 3-10). New York: Oxford University Press.

Sagiv, N. (2008). Re-conceptualising synaesthesia: Empirical motivation and theoretical advantages. 7th Meeting of the American Synesthesia Association, McMaster University, Hamilton, Ontario.

Sagiv., N. \& Ben-Tal, O. (submitted). Nocturnal synesthesia.

Sagiv, N., Dean, R.T., \& Bailes, F. (2009). Algorithmic Synaesthesia. In Dean RT (Ed.), The Oxford Handbook of Computer Music (pp 294-311). New York: Oxford University Press.

Sagiv, N., Heer, J., Robertson, L. (2006). Does binding of synesthetic color to the evoking grapheme require attention? Cortex, 42, 232-242.

Sagiv, N., Simner, J., Collins, J., Butterworth, B., \& Ward, J. (2006). What is the relationship between synaesthesia and visuo-spatial number forms? Cognition, 101, 114-128.

Sagiv, N., \& Ward, J. (2006). Cross-modal interactions: Lessons from synesthesia. Progress in Brain Research, 155, 263-275.

Shanon, B. (1982). Colour associates to semantic linear orders. Psychological Research, 44, 75-83.

Shanon, B. (2003). Hallucinations. Journal of Consciousness Studies, 10, 3-31.

Simner, J. (in press). Defining Synaesthesia. British Journal of Psychology. DOI: 10.1348/000712610X528305.

Simner, J., \& Holenstein, E. (2007). Ordinal linguistic personification: The systematic attribution of animate qualities to the linguistic units of ordinal sequences. Journal of Cognitive Neuroscience, 19, 694-703.

Simner, J., \& Hubbard, E.M. (2006). Variants of synaesthesia interact in cognitive tasks: Evidence for implicit associations and late connectivity in cross-talk theories. Neuroscience, 143, 805-14.

Simner, J., Mulvenna, C., Sagiv, N., Tsakanikos, E., Witherby, S.A., Fraser, C., Scott, K., \& Ward, J. (2006). Synaesthesia: The prevalence of atypical cross-modal experiences. Perception, 35, 1024-1033.

Slade, P. \& Bentall, R. (1988). Sensory Deception: A scientific Analysis of Hallucinations. London: Croom-Helm.

Smilek, D., Malcolmson, K.A., Carriere, J.S., Eller, M., Kwan, D., \& Reynolds, M. (2007). When ' 3 "' is a Jerk and " $E$ ', is a King: Personifying Inanimate Objects in Synesthesia. Journal of Cognitive Neuroscience, 19, 981-992.

Spiller, M.J., \& Jansari A.S. (2008). Mental imagery and synaesthesia: Is synaesthesia from internally-generated stimuli possible? Cognition, 109, 143-51.

Tang, J., Ward, J. \& Butterworth, B. (2008). Number forms in the brain. Journal of Cognitive Neuroscience, 20, 1547-1556.

Taylor, D.C., \& Lochery, M. (1987). Temporal lobe epilepsy: origin and significance of simple and complex auras. Journal of Neurology Neurosurgery ad Psychiatry, 50, 673-81.

Van Campen, C. (2007). The Hidden Sense: Synesthesia in Art and Science. Cambridge, MA: MIT Press. 
Vihvelin, H. (1948). On the differentiation of some typical forms of hypnagogic hallucinations. Acta Psychiatrica Scandinavica, 23, 359-389.

Ward, J. (2008). The Frog Who Croaked Blue. Oxford: Routledge.

Ward, J., Li, R., Salih, S., \& Sagiv, N. (2007). Varieties of Grapheme-Colour Synaesthesia: A New Theory of Phenomenological and Behavioural Differences. Consciousness and Cognition, 16, 913-931.

Ward, J., \& Sagiv, N. (2007). Synaesthesia for Finger Counting and Dice Patterns: A Case of Higher Synaesthesia? Neurocase, 13, 86-93.

Zamboni, G., Budriesi, C., \& Nichelli, P. (2005). "Seeing oneself": a case of autoscopy. Neurocase, 11, 212-5. 